\title{
Thrombectomy or intravenous thrombolysis in patients with NIHSS of 5 or less?
}

\author{
Valerio Da Ros ${ }^{\mathrm{a}, \mathrm{b}, *}$, Jonathan Cortese ${ }^{\mathrm{b}}$, Olivier Chassin ${ }^{\mathrm{c}}$, Aymeric Rouchaud $^{\mathrm{b}}$, \\ Mariana Sarov $^{\mathrm{c}}$, Jildaz Caroff ${ }^{\mathrm{b}}$, Cristian Mihalea $^{\mathrm{a}, \mathrm{b}}$, Silvia Minosse ${ }^{\mathrm{a}}$, Irina Taifas ${ }^{\mathrm{c}}$, \\ Jacopo Scaggiante ${ }^{a}$, Laura Greco ${ }^{a}$, Leon Ikka ${ }^{\mathrm{b}}$, Nidhal Ben Achour ${ }^{\mathrm{b}}$, \\ Francesca Di Giuliano ${ }^{\mathrm{a}}$, Augustin Ozanne ${ }^{\mathrm{b}}$, Nicolas Legris ${ }^{\mathrm{c}}$, Marina Diomedi $^{\mathrm{d}}$, \\ Fabrizio Sallustio $^{\mathrm{d}}$, Roberto Floris ${ }^{\mathrm{a}}$, Christian Denier ${ }^{\mathrm{c}}$, Laurent Spelle ${ }^{\mathrm{b}}$ \\ a Department of diagnostic imaging and interventional radiology, "Tor Vergata" university Hospital, viale Oxford, 81, 00133 Rome, Italy \\ b Interventional Neuroradiology NEURI Center, Hôpital Bicêtre, 78, rue du Général-Leclerc, 94270 Le Kremlin Bicêtre, France \\ c Department of Neurology, Bicêtre Hospital, 78, rue du Général-Leclerc, 94270 Le Kremlin-Bicêtre, Paris, France \\ d Department of Neurology, Stroke Unit, "Tor Vergata" University Hospital, viale Oxford, 81, 00133 Rome, Italy
}

\section{A R T I C L E I N F O}

Available online xxx

\begin{abstract}
A B S T R A C T
Background and purpose. - To compare outcomes of minor stroke patients with intracranial vessel occlusions (IVO) underwent mechanical thrombectomy (MT) versus those treated with intravenous thrombolysis alone (IVT).

Methods. - We retrospectively reviewed two large prospective stroke databases from two European centers searching for patients admitted with minor stroke (i.e. NIHSS Score $\leq 5$ ), baseline $\mathrm{mRS}=0$ and occlusion of the M1-M2 segment of the middle cerebral artery (MCA). Groups receiving (A) IVT alone and (B) MT+/-IVT were compared. Primary outcome measures were MT safety, successful recanalization rate (mTICI 2b-3) and NIHSS shift (discharge NIHSS minus admission NIHSS); secondary outcomes included discharge rates and excellent outcome (mRS 0-1) at 3 months. Univariate and multivariate analyses were performed.

Results. - Thirty-two patients were enrolled in Group B (19 MT alone; 13 MT + IVT) and 24 in Group A. Successful recanalization (mTICI $2 \mathrm{~b}-3$ ) was obtained in $100 \%$ of cases in Group B vs $38 \%$ in Group A. Symptomatic hemorrhagic transformation rate did not differ between the two groups. Multivariate analysis reported MT as the only predictor of early $(<12 \mathrm{~h})$ favorable NIHSS shift and lower NIHSS at discharge. Moreover, discharge at home and excellent outcome at 3-month follow-up were statistically associated with MT.

Conclusions. - MT in patients with minor strokes and intracranial vessel occlusion (IVO) is safe and can determine a rapid improvement of NIHSS Score. MT seems also associated with a higher rate of patients discharged at home after hospitalization and better clinical outcome at 3-month follow-up. Larger randomized trials are warranted to confirm these results.
\end{abstract}

(C) 2019 Elsevier Masson SAS. All rights reserved.

\section{Background and purpose}

Ischemic strokes with mild symptoms have been associated with a good prognosis [1].

Only $5.5 \%$ of patients with a minor stroke, experience a clinical neurological deterioration, even when no intravenous thrombolysis (IVT) is provided [2]. However, the presence of intracranial

\footnotetext{
* Corresponding author at: Interventional Neuroradiology Division, University of Rome Tor Vergata, viale Oxford, 81, Rome, 00133 Italy.

E-mail address: darosvalerio@gmail.com (V. Da Ros).
}

vessel occlusion (IVO) increases the risk of early deterioration and poor outcomes, despite the initial low neurological symptoms (i.e. NIHSS Score $\leq 5$ ) [3]. Indeed, up to $15 \%$ of these patients will experience an unstable course in the first 24 hours with 90-day significant disability rates [4].

Vessel recanalization is crucial to achieve a good clinical outcome and IVT has been shown to yield better outcomes compared with no treatment $[3,5,6]$. Anyway, some patients may be not eligible to IVT or extended thrombus burden may reduce its effect [7].

Recently, mechanical thrombectomy (MT) was defined as the standard of care for patients with IVO and severe strokes [8]. How- 
ever, outcomes for patients with low National Institutes of Health Stroke Scale (NIHSS) Scores come from only two $[9,10]$ of the five seminal thrombectomy trials [11,12,13], with only a small number of patients enrolled.

Thus, the right indication for MT in patients with minor stroke [14] as well as MT beneficial effect compared with IVT remains unclear and underrepresented in existing studies.

In this study, we report the clinical outcomes of minor stroke patients with IVO comparing MT with a historical group of patients treated, before the advent of MT at our institutions, with IVT alone.

\section{Methods}

\section{Outcome definition and measurements}

Minor stroke was defined by NIHSS Score of $\leq 5[4]$.

IVO was defined as an intracranial arterial occlusion involving the M1 and proximal M2 segment of the middle cerebral artery, diagnosed using magnetic resonance imaging (MRI) or computed tomography (CT) angiography.

Neurological deterioration (ND) [15,16] was defined as an increase in the NIHSS by $\geq 4$ points compared to baseline, or as the onset of new neurological symptoms whereby a subcomponent of the NIHSS Scale that was previously given a score of 0 was subsequently given a score of 1 or more points.

Neurological fluctuation (NF) was defined by $\geq 4$ points change in NIHSS Score in the 24 hours after the onset of minor stroke symptoms and prior to MT.

Successful recanalization by MT was defined by a final modified thrombolysis in cerebral infarction (mTICI) grade of $2 \mathrm{~b}$ or 3 , according to Zaidat et al. [17].

Hemorrhagic transformation (HT) was graded based on the ECASS II classification [18]. Symptomatic HT was defined as an intracerebral hemorrhage with an increase in the NIHSS Score of $\geq 4$ points (HT-2). Asymptomatic HT was defined as an intracerebral hemorrhage with an increase in the NIHSS Score of $\leqslant 4$ points (HT-1).

MT complications included: embolism in a new vascular territory, vasospasm, and femoral artery access injuries.

\section{Clinical assessment}

Baseline demographics and medical history were collected from our prospective stroke registries and comprised a neurological examination performed at admission by a neurologist in the emergency department, including NIHSS Score assessment and onset-to-treatment times recording.

NIHSS Score was evaluated at admission, at 12 hours, whenever the neurological symptoms changed, 24 hours after the MT, and daily until patient discharge. MT alone was performed in case of contraindications for IVT or as a rescue therapy after IVT.

NIHSS shift was defined as a change in NIHSS Score between two assessments.

Early neurological improvement (ENI) was defined as an NIHSS Score of 0 to 1 after 24-hour from MT [19].

Follow-up, including the modified Rankin Scale Score (mRS), took place during an inpatient visit after three months or via a structured telephone interview performed by a neurologist not blinded to the type of treatment.

A mRS Score of 0-1 at 90 days was defined as being indicative of excellent outcomes; a mRS Score of 2 at 90 days was defined as good outcomes.

\section{Patients selection}

We retrospectively reviewed two large prospective stroke datasets from two comprehensive stroke centers.
Demographic data, pre-stroke mRS Score, baseline NIHSS, occluded vessel site, daily NIHSS during hospitalization and at discharge, as well as the discharge mRS Score, discharge location (home or rehabilitation) and the 90 days mRS were collected.

Two groups were identified and compared: the medical group (Group A) and the interventional group (Group B).

In the Group A we included all consecutive patients admitted with minor stroke and IVO, demonstrated by imaging between September 2014 and September 2015, treated with IVT alone.

The interventional group (Group B) included all consecutive patients treated with MT alone or in association with IVT, admitted to the two centers involved in this study, between September 2015 and July 2017 with a diagnosis of acute ischemic stroke (within $24 \mathrm{~h}$ ), an initial NIHSS Scores $\leq 5$ and an evidence of an IVO, candidate to MT due to M1-M2 segment occlusion of the middle cerebral artery. Patients were selected for MT if rt-PA was not effective or contraindicated, and/or in case of neurological fluctuation. Moreover, patients were treated with MT after rt-PA in case of new somatosensory symptoms.

In both groups, intravenous rt-PA was prescribed within 4.5 hours of onset according to guidelines [20] and patients were treated only with an ASPECT [21] Score > 7 .

We excluded patients with modified Rankin Scale Scores $\geq 1$ prior to the MT so as to better understand the efficacy of MT in this subgroup of ischemic stroke patients.

\section{Imaging studies}

Patients with suspected acute ischemic stroke underwent a standardized stroke imaging protocol either with MRI (including axial DWI, 3D time-of-flight MR-angiography of the intracranial vessels, axial FLAIR, and axial gradient echo (GRE) imaging) or three-phasic angio-CT $\pm \mathrm{CT}$-perfusion imaging.

For the interventional group (Group B) the occlusion site was confirmed by digital subtraction angiography (DSA).

Cerebral infarction and post-treatment intracranial hemorrhage were assessed with non-enhanced CT scan performed 24 hours after treatment, or sooner in case of clinical deterioration.

\section{Stroke therapy}

In the interventional group (Group B), IVT was administered as a bridging therapy to all patients when eligible. In case of wake-up stroke, the patient's eligibility for MT was based on imaging criteria (e.g. CT-perfusion and/or DWI-FLAIR mismatch) [19,22].

Indication for MT was discussed by a multidisciplinary team (neurologist and neuro-interventionist) and assessed on an individual basis according to the patient's comorbidities, pre-stroke condition and common signs of neurological impairment (e.g. aphasia, neglect).

No age limit was defined. MT was performed under conscious sedation for all of the patients.

The type of endovascular technique (e.g. stent-retriever or aspiration) was at the discretion of the treating physician. The devices types used for MT, the number maneuvers, and the intra-procedural complications were assessed. Successful reperfusion was based on use of the modified Thrombolysis in Cerebral Infarction (mTICI) Scale $[17,23]$.

\section{Statistical analysis}

Median and interquartile range (IQR) of all continuous variables were calculated for the entire patient population. Categorical variables were reported as proportions.

The Mann-Whitney rank-sum test for independent samples was performed to compare the NIHSS parameters in the two 


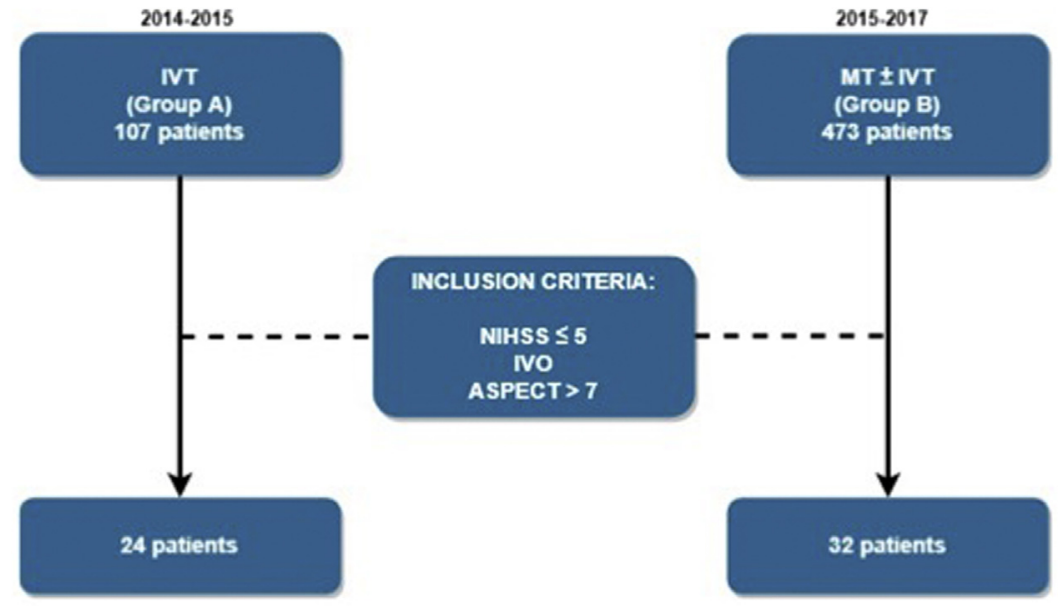

Fig. 1. : Flow chart of patient inclusion and treatment allocation. IVT: intravenous thrombolysis; MT: mechanical thrombectomy.

Table 1

Characteristics of the Patients at Baseline.

\begin{tabular}{lll}
\hline Variables & $\begin{array}{l}\text { Thrombectomy Group } \\
(n=29)\end{array}$ & $\begin{array}{l}\text { IVT Group } \\
(n=24)\end{array}$ \\
\hline Age, yrs (range) & $70(23-92)$ & $68(40-95)$ \\
Male sex, $n(\%)$ & $13(45)$ & $15(63)$ \\
Atrial fibrillation, $n(\%)$ & $10(34)$ & $4(17)$ \\
Diabetes mellitus, $n(\%)$ & $1(3)$ & $6(25)$ \\
Hypertension, $n(\%)$ & $22(76)$ & $17(71)$ \\
Smoking, $n$ (\%) & $4(14)$ & $9(38)$ \\
Hypercholesterolemia & $11(38)$ & $12(50)$ \\
Baseline NIHSS Score, Mean (SD) & $4(2-4)$ & $4(3.5-5)$ \\
Imaging study & & $0(0)$ \\
RM & $21(72)$ & $0(0)$ \\
CT-Perfusion & $6(21)$ & $24(100)$ \\
CT-Angiography & $11(38)$ & $0(0)$ \\
Occlusion site, $n(\%)$ & $12(41)$ & $24(100)$ \\
M1 & $13(45)$ & $0(0)$ \\
M2 & $4(14)$ & $9(38)$ \\
M1 + ICA & $15(52)$ & $13(54)$ \\
Toast & $8(28)$ & $2(8)$ \\
Cardioembolic & $6(20)$ & \\
Large artery & & \\
Undetermined & &
\end{tabular}

groups (Group A and Group B). To compare NIHSS onset and NIHSS (12 hours, 24 hours, discharge), the Wilcoxon test for paired samples was performed.

For categorical data Fisher's exact test was used. The significance level was set at 0.05 .

\section{Results}

During the study period, 32 patients of the 473 patients treated with Mechanical Thrombectomy (MT) did meet the inclusion criteria and were enrolled in the interventional arm (Group B), while 24 of 107 patients treated with IVT alone were included in the medical management arm (Group A). Fig. 1.

The primary analysis demonstrated similar demographics and baseline characteristics between Group A and Group B.

Demographic information, medical history, onset NIHSS and imaging modalities of both groups are presented in Table 1.

All patients of Group A presented with M2 segment IVO on initial imaging (10 on the right-side; 14 on the left-side). In the medical group, IVT was administered with a mean onset to needle delay of $170 \pm 88 \mathrm{~min}$.

In Group B, IVO site on initial imaging were: M1 segment in 13 patients ( 7 on the right-side; 6 on the left-side); M2 segment in 15 patients ( 6 on the right-side; 9 on the left-side); internal carotid artery and M1 segment occlusion in 4 patients ( 3 on the right-side; 1 on the left-side).

In Group B, the occlusion site on CT or MR imaging was confirmed at the next DSA in 29/32 patients (91\%). In three patients (9\%), vessel recanalization was observed with DSA (two TICI 2b; one TICI 3) and no MT was performed; all three patients presented with an initial M2 occlusion on MRI and received IVT; in one of them an asymptomatic HT type- 1 was observed. These patients were excluded from the final comparative analysis.

4 of the 32 patients (13\%) exhibited NF. For these patients, the mean onset time to femoral puncture was $761 \pm 227 \mathrm{~min}$. In all patients with NF, symptoms resolved after a successful MT recanalization.

Overall, in Group B, 19/32 patients (59\%) received IVT + MT [18 (95\%) as a bridging therapy with a mean onset to femoral puncture delay of $237 \pm 78 \mathrm{~min}$; one $(5 \%)$ as a rescue therapy due to IVT failure. In this case, IVT was administered 185 min after the onset of symptoms. Due to a subsequent ND, a second MRI imaging evaluation was performed and a second IVT was administered as a bridging therapy to MT (delay between IVTs was $297 \mathrm{~min}$, IVT-to-MT delay was $18 \mathrm{~min})$.

$13 / 32$ patients (40\%) underwent MT alone [10 (77\%) due to IVT contraindication and 3 (33\%) due to wake-up strokes with positive FLAIR on MRI)].

For patients treated with MT alone, the mean door-to-needle time was $230 \pm 20 \mathrm{~min}$.

MT was performed with a stent-retriever and proximal balloon catheter aspiration in 15/32 (47\%) patients and 14/32 (43\%) were treated by primary aspiration thrombectomy. The mean number of recanalization passages was 1.7 (median: 1 ).

Successful IVO recanalization was achieved for all of the treated patients (100\%) in Group B (24 TICI 3; 5 TICI 2b) and in 9/24 patients $(38 \%)$ in Group A (38\%) $(P<0.001)$.

NIHSS at the onset was 4 (range 2-4) for Group B and of 4 (3.5-5) for Group A $(P=0.106)$.

NIHSS shift at 12 h was $1(0-2.25)$ for Group B and 4 (2-5) for Group A $(P=0.005)$; NIHSS shift at 24 h was $1(0-2)$ for Group B and of $3(1-4)$ for Group A $(P=0.024)$; NIHSS shift at hospital discharge was $1(0-2)$ for Group B and of $2(1-3.5)$ for Group A $(P=0.036)$ (Table 2).

The mean hospitalization time was 5.6 days (median: 4 days). In Group B, 23/29 (79\%) patients were discharged at home while $13 / 24$ patients in Group A (54\%). The remaining patients were discharged to rehabilitation in both groups (Fig.2).

In Group B, 3 patients experience an $\mathrm{HI}-1$ (9\%) and 2 patients experienced an HT-2 (6\%): the first patient exhibited a tight stenosis 


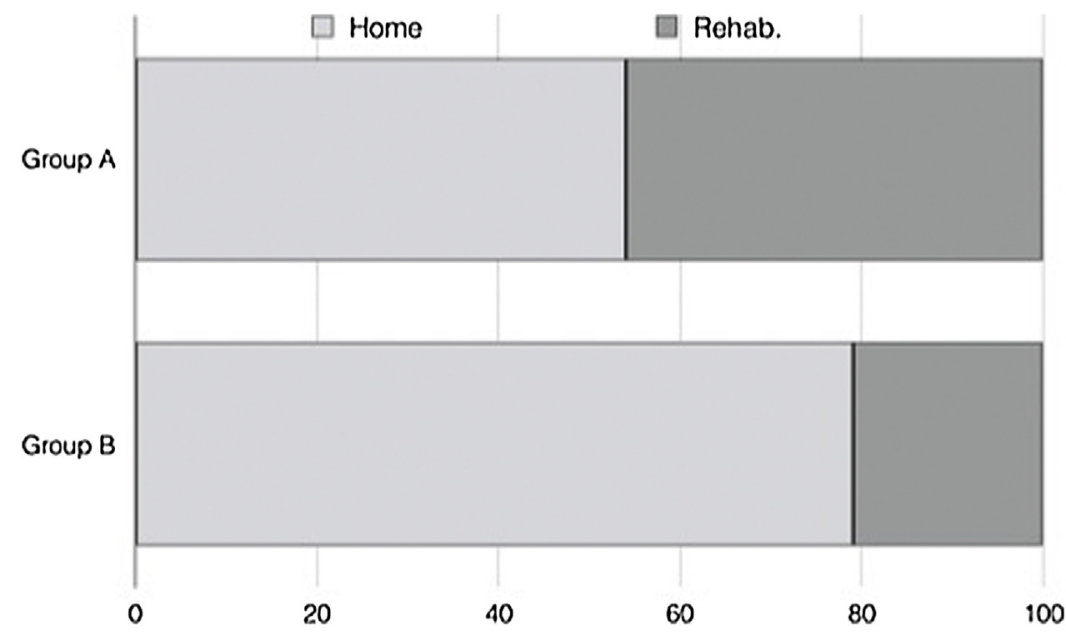

Fig. 2. : Hospital discharge location. Group A: alone; Group B: mechanical thrombectomy +/- intravenous thrombolysis.

Table 2

Clinical outcomes.

\begin{tabular}{llll}
\hline Median NIHSS (IQR) & Group A $(n=24)$ & Group B $(n=29)$ & $P$ value \\
\hline Onset $^{\mathrm{a}}$ & $4(3.5-5)$ & $4(2-4)$ & 0.106 \\
$12 \mathrm{~h}^{\mathrm{a}}$ & $4(2-5)$ & $1(0-2.25)$ & 0.005 \\
$24 \mathrm{~h}^{\mathrm{a}}$ & $3(1-4)$ & $1(0-2)$ & 0.024 \\
Discharge $^{\mathrm{a}}$ & $2(1-3.5)$ & $1(0-2)$ & 0.036 \\
mRS 0-1 $^{\mathrm{b}}$ & $11(45.80 \%)$ & $27(93.10 \%)$ & $<0.001$ \\
mRS 0-2 & $19(79.17 \%)$ & $27(93.10 \%)$ & 0.223 \\
\hline
\end{tabular}

IQR: interquantile range; $n$ : number of patients.

a Mann-Whitney test.

b Fisher test.

of the internal carotid artery associated with left-side M1 IVO and undergone MT alone due to IVT contraindication. A successful TICI 3 recanalization of the left-side $M 1$ segment was achieved, and the tight stenosis was initially not treated; anyway after 525 minutes from the procedure, new neurological symptoms appeared and a left internal carotid artery stenting was attempted unsuccessfully due to failure of crossing the carotid bulb occlusion. The patient experienced a symptomatic hemorrhagic transformation (HI-2) at 24 hours, with a NIHSS increase of 13 points at discharge and presented a mRS of 2 at 90 days follow-up. The second patient with HI-2 (86 years old lady) presented with right M1 occlusion, underwent IVT + MT and experienced a 24 h symptomatic hemorrhagic transformation (HI-2), with a 15-point increase in NIHSS Score at discharge and a mRS of 6 at three month follow-up.

In Group A, 3 patients experience a HI-1 (13\%) and 1 patient reported a $\mathrm{HI}-2(4 \%)$ at 24 hours, with a 12 point increase in their NIHSS Score at discharge and a mRS of 4 at 3-month follow-up

Overall, an excellent outcome (i.e. mRS 0-1) at three months was obtained in $27 / 29$ patients (93\%) in Group B and $11 / 24$ in Group A $(45 \%)(P<0.001)$. A good outcome (mRS $0-2)$ was observed in $27 / 29$ patients (93\%) in Group B and in 19/24 (72\%) patients in Group A $(P<0.223)$ (Fig.3).

\section{Discussion}

Our study demonstrated that mechanical thrombectomy is safe and effective in increasing chance of successful vessel recanalization (i.e. mTICI $2 b-3$ ) in patients with minor strokes due to IVO, obtaining a more favorable NIHSS shift at hospital discharge and an excellent outcome (mRS 0-1), as compared to medical therapy alone, without higher complication rates.

Nowadays, the best management for minor strokes still remained unclear and unstandardized.
Most patients admitted with a diagnosis of minor stroke are treated with IVT alone and several others are still excluded from IVT because considered doing clinically too good to be treated [2].

Moreover, results of the pooled meta-analysis data of recent randomized trials indicate that no benefit of MT could be statistically demonstrated for patients with a NIHSS Score of $<10$ [24].

Therefore, MT in patients with NIHSS $\leq 5$ and IVO remains described only in small case series [8].

Anyway, despite the mild clinical onset, vessels' recanalization seems necessary to obtain a good outcome also for minor strokes $[3,5,6]$ because, when no acute recanalization is achieved, about one third of minor stroke patients could not ambulate independently at hospital discharge [25] and are more prone to experience neurological deterioration with poor clinical outcomes at 90 day follow-up $[26,27,28]$.

Our study confirmed that the better NIHSS shift observed was independently related with both the endovascular intervention and the higher recanalization rate obtained with MT compared with medical treatment.

Even if reassuring efficacy results of medical treatment for acute mild ischemic stroke patients are well described in larger series, with good outcomes obtained in more than $80 \%$ of the cases, the main concern of standalone IVT treatment is the frequent lack of information regarding the presence of IVO for most of the studied patients [29].

Despite mild stroke onset, when IVO is documented on imaging, medical therapy demonstrated its inefficacy in more than $40 \%$ of cases, with subsequent neurological deterioration [8] and high disability rates [30].

In our study, IVO was confirmed by imaging in both groups and results in terms of IVT recanalization rates were in line with those presents in literature, confirming the reduced IVT efficacy to obtain a successful recanalization in case of IVO in a significant number of patients. Moreover, in this series, about $40 \%$ of the patients of Group $\mathrm{B}$ had contraindications to thrombolysis. Hence, MT was often the only available treatment for an acute IVO recanalization.

To the best of our knowledge, at the moment there is only one prospective study and few reports, comparing medical therapy with endovascular intervention in minor stroke [8,31].

However, all the existing studies comparing MT versus IVT, in patients with NIHSS Scores of 5 or less, are based on limited number of patients enrolled for comparison once the groups are adequately matched. Although also our study did not overcome this issue for comparison of the two treatments modalities, it seems to confirm not only the overall improved clinical outcome in minor stroke patients treated with MT. In addition, it also demonstrated the 


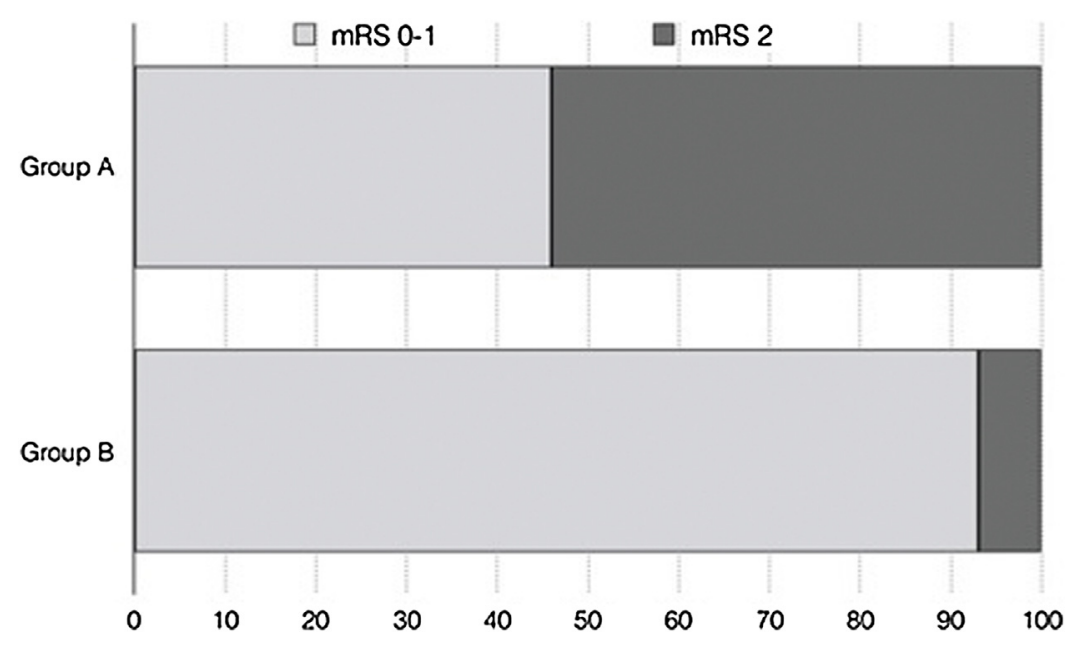

Fig. 3. : 3-months excellent (0-1) and good (2) mRS outcomes. Group A: alone; Group B: mechanical thrombectomy +/- intravenous thrombolysis.

ability of MT to determine a very early neurological improvement $(<12 \mathrm{~h})$ as compared to medical therapy. Precisely, despite the 90days good outcome (mRS 0-2) was achieved in both groups in our comparative analysis, the rapid clinical improvement observed in Group B patients was associated with a higher chance of being discharged at home with an excellent outcome (mRS 0-1) at 90-day follow-up.

These results may have potential benefit also in term of costefficacy because we reported a lower number of in-hospital days for Group B patients, without needing further rehabilitation periods. Moreover, MT seemed determinant as rescue therapy for those patients experiencing neurological deterioration or long lasting neurological fluctuation despite previous IVT administration. Indeed, the slow progression of NF symptoms, despite previous IVT administration, was the consequence of good leptomeningeal collaterals. However, over time the activation of the cerebral blood reserve, including vessels' vasodilatory capacity and vasoreactivity, progressively decline and most of the time ends with a severe cerebral blood "steal" phenomenon, followed by neurological deterioration [28]. Thus, in case of IVO and long lasting symptoms or striking progression but negative FLAIR imaging on MR and/or no lesions on cerebral blood flow maps, MT was considered a good indication despite the late time from clinical onset, disproving the time window dogma for endovascular treatment, that is recently emphasized in the DAWN trial results [32].

\section{Limitations}

Unfortunately, despite the two groups presented an excellent matching, this study has several limitations. First, although the prospective data collection, this study was based on a retrospective analysis. Secondly, the limited time during which the study was conducted by the two involved tertiary stroke centers was mainly responsible for the small sample size for comparison. Third, different treatments and imaging modalities were used. Indeed, the interventions were at the physician's discretion and imaging modalities were based on MRI or CT institution protocols (i.e. potential selection bias). Moreover, outcome assessments were not blinded, introducing a potential assessment bias.

Anyway, although the retrospective study, each neurological assessment was carefully obtained by independent examinations.

Moreover, it should be noted that the medical groups (Group A) derived from a different study period. However, the homogeneous medical therapy, treating patients with the same protocol, reduced the possibility of residual confounding. On the other hand, the MT cohort was treated with contemporary techniques and should be representative of the current real-world practice.

Fourth, a significant proportion of patients in the interventional arm was treated after clinical deterioration or after long lasting symptoms with neurological fluctuation, attempting a rescue MT after IVT failure. Therefore, theoretically clinical outcome could be improved, performing thrombectomy without delay.

Finally, Group A included only patients with M2 segment IVO. Anyway, IVT results may be even lower adding larger segment occlusions to the analysis.

\section{Conclusions}

MT in patients with minor strokes and IVO appears safe and effective to determine a more rapid NIHSS improvement as compared with medical treatment. MT seems also associated with a higher rate of patient discharge to home after hospitalization with excellent outcome at 3-month follow-up and even cost-efficacy benefits. Larger randomized prospective trials are warranted to confirm these results.

\section{Ethics statement}

This study was approved by the Institutional Review Board (IRB) of the Bicetre University Hospital. Written informed consent was not obtained from participants due to the study design. The hospital IRB, therefore, waived the need for written informed consent from the participants.

\section{Disclosure of interest}

The authors declare that they have no competing interest.

\section{References}

[1] Smith EE, Fonarow GC, Reeves MJ, Cox M, Olson DM, Hernandez AF, et al. Outcomes in mild or rapidly improving stroke not treated with intravenous recombinant tissue-type plasminogen activator: findings from get with the guidelines-stroke. Stroke 2011;42:3110-5.

[2] Willey JZ, Stillman J, Rivolta JA, Vieira J, Doyle MM, Linares G, et al. Too good to treat? Outcomes in patients not receiving thrombolysis due to mild deficits or rapidly improving symptoms. Int J Stroke 2012;7:202-6.

[3] Rajajee V, Kidwell C, Starkman S, Ovbiagele B, Alger JR, Villablanca P, et al, Early MRI and outcomes of untreated patients with mild or improving ischemic stroke. Neurology 2006;67:980-4.

[4] Khatri P, Conaway MR, Johnston KC. Acute Stroke Accurate Prediction Study (ASAP) Investigators. Ninety-day outcome rates of a prospective cohort of consecutive patients with mild ischemic stroke. Stroke 2012;43:560-2. 
[5] IST-3 collaborative groupSandercock P, Wardlaw JM, Lindley RI, Dennis M, Cohen $\mathrm{G}$, et al. The benefits and harms of intravenous thrombolysis with recombinant tissue plasminogen activator within $6 \mathrm{~h}$ of acute ischaemic stroke (the third international stroke trial [IST-3]): a randomised controlled trial. Lancet 2012;379:2352-63.

[6] Smith EE, Abdullah AR, Petkovska I, Rosenthal E, Koroshetz WJ, Schwamm LH. Poor outcomes in patients who do not receive intravenous tissue plasminogen activator because of mild or improving ischemic stroke. Stroke 2005;36:2497-9.

[7] Behrens L, Möhlenbruch M, Stampfl S, Ringleb PA, Hametner C, Kellert L, et al. Effect of thrombus size on recanalization by bridging intravenous thrombolysis. Eur J Neurol 2014;21:1406-10.

[8] Haussen DC, Bouslama M, Grossberg JA, Anderson A, Belagage S, Frankel M, et al. Too good to intervene? Thrombectomy for large vessel occlusion strokes with minimal symptoms: an intention-to-treat analysis. J Neurointerv Surg 2017:9:917-21.

[9] Berkhemer OA, Fransen PSS, Beumer D, van den Berg LA, Lingsma HF, Yoo AJ, et al. A randomized trial of intraarterial treatment for acute ischemic stroke. $\mathrm{N}$ Engl J Med 2015;372:11-20.

[10] Campbell BCV, Mitchell PJ, Kleinig TJ, Dewey HM, Churilov L, Yassi N, et al. Endovascular therapy for ischemic stroke with perfusion-imaging selection. N Engl J Med 2015;372:1009-18.

[11] Goyal M, Demchuk AM, Menon BK, Eesa M, Rempel JL, Thornton J, et al. Randomized assessment of rapid endovascular treatment of ischemic stroke. $\mathrm{N}$ Engl J Med 2015;372:1019-30.

[12] Jovin TG, Chamorro A, Cobo E, de Miquel MA, Molina CA, Rovira A, et al. Thrombectomy within 8 hours after symptom onset in ischemic stroke. N Engl J Med 2015;372:2296-306.

[13] SaverJL, Goyal M, Bonafe A, Diener H-C, Levy EI, Pereira VM, et al. Stent-retriever thrombectomy after intravenous t-PA vs. t-PA alone in stroke. N Engl J Med 2015;372:2285-95.

[14] Powers WJ, Derdeyn CP, Biller J, Coffey CS, Hoh BL, Jauch EC, et al, 2015 American Heart association/American Stroke association focused update of the 2013 guidelines for the early management of patients with acute ischemic stroke regarding endovascular treatment: a guideline for healthcare professionals from the American Heart association/American stroke association. Stroke 2015;46:3020-35.

[15] Kwan J, Hand P. Early neurological deterioration in acute stroke: clinical characteristics and impact on outcome. OJM 2006:99:625-33.

[16] Ois A, Martinez-Rodriguez JE, Munteis E, Gomis M, Rodríguez-Campello A, Jimenez-Conde J, et al. Steno-occlusive arterial disease and early neurological deterioration in acute ischemic stroke. Cerebrovasc Dis 2008;25:151-6.

[17] Zaidat OO, Yoo AJ, Khatri P, Tomsick TA, von Kummer R, Saver JL, et al. Recommendations on angiographic revascularization grading standards for acute ischemic stroke: a consensus statement. Stroke 2013;44:2650-63.

[18] Molina CA, Alvarez-Sabín J, Montaner J, Abilleira S, Arenillas JF, Coscojuela P, et al. Thrombolysis-related hemorrhagic infarction: a marker of early reperfusion, reduced infarct size, and improved outcome in patients with proximal middle cerebral artery occlusion. Stroke 2002;33:1551-6.
[19] Kharitonova T, Mikulik R, Roine RO, Soinne L, Ahmed N, Wahlgren N, et al. Association of early national institutes of health stroke scale improvement with vessel recanalization and functional outcome after intravenous thrombolysis in ischemic stroke. Stroke 2011:42:1638-43.

[20] Jauch EC, Saver JL, Adams Jr HP, Bruno A, Connors JJB, Demaerschalk BM, et al. Guidelines for the early management of patients with acute ischemic stroke: a guideline for healthcare professionals from the American Heart Association/American Stroke Association. Stroke 2013;44:870-947.

[21] Barber PA, Demchuk AM, Zhang J, Buchan AM. Validity and reliability of a quantitative computed tomography score in predicting outcome of hyperacute stroke before thrombolytic therapy. ASPECTS Study Group. Alberta Stroke Programme Early CT Score. Lancet 2000;355:1670-4.

[22] Madai VI, Wood CN, Galinovic I, Grittner U, Piper SK, Revankar GS, et al Clinical-radiological parameters improve the prediction of the thrombolysis time window by both mri signal intensities and DWI-FLAIR mismatch. Cerebrovasc Dis 2016;42:57-65.

[23] Fugate JE, Klunder AM, Kallmes DF. What is meant by "TICI"? AJNR Am J Neuroradiol 2013:34:1792-7.

[24] Goyal M, Menon BK, van Zwam WH, Dippel DWJ, Mitchell PJ, Demchuk AM, et al. Endovascular thrombectomy after large-vessel ischaemic stroke: a meta-analysis of individual patient data from five randomised trials. Lancet 2016:387:1723-31.

[25] Romano JG, Smith EE, Liang L, Gardener H, Camp S, Shuey L, et al. Outcomes in mild acute ischemic stroke treated with intravenous thrombolysis: a retrospective analysis of the Get with the guidelines-stroke registry. JAMA Neurol 2015;72:423-31.

[26] Zhu W, Churilov L, Campbell BCV, Lin M, Liu X, Davis SM, et al. Does large vessel occlusion affect clinical outcome in stroke with mild neurologic deficits after intravenous thrombolysis? J Stroke Cerebrovasc Dis 2014;23:2888-93.

[27] Smith WS, Tsao JW, Billings ME, Johnston SC, Hemphill 3rd JC, Bonovich DC, et al. Prognostic significance of angiographically confirmed large vessel intracranial occlusion in patients presenting with acute brain ischemia. Neurocrit Care 2006;4:14-7.

[28] Smith WS, Lev MH, English JD, Camargo EC, Chou M, Johnston SC, et al. Significance of large vessel intracranial occlusion causing acute ischemic stroke and TIA. Stroke 2009;40:3834-40.

[29] Strbian D, Piironen K, Meretoja A, Sairanen T, Putaala J, Tiainen M, et al. Intravenous thrombolysis for acute ischemic stroke patients presenting with mild symptoms. Int J Stroke 2013;8:293-9.

[30] Kim J-T, Heo S-H, Yoon W, Choi K-H, Park M-S, Saver JL, et al. Clinical outcomes of patients with acute minor stroke receiving rescue IA therapy following early neurological deterioration. J Neurointerv Surg 2016;8:461-5.

[31] Haussen DC, Lima FO, Bouslama M, Grossberg JA, Silva GS, Lev MH, et al. Thrombectomy versus medical management for large vessel occlusion strokes with minimal symptoms: an analysis from STOPStroke and GESTOR cohorts. J Neurointerv Surg 2018:10:325-9.

[32] Nogueira RG, Jadhav AP, Haussen DC, Bonafe A, Budzik RF, Bhuva P, et al. Thrombectomy 6 to 24 hours after stroke with a mismatch between deficit and infarct. N Engl J Med 2017, http://dx.doi.org/10.1056/NEJMoa1706442. 